\title{
CORRESPONDENCE
}

\section{Comment on: "Overcoming barriers in access to ophthalmic education with virtual learning"}

(c) The Author(s), under exclusive licence to The Royal College of Ophthalmologists 2021

Eye (2022) 36:1703-1704; https://doi.org/10.1038/s41433-021-01769-7

\section{TO THE EDITOR:}

We appreciated the article titled "Overcoming barriers in access to ophthalmic education with virtual learning" by He et al. [1]. They highlighted the importance of virtual formats in ophthalmic education, the good acceptance of webinars among medical students, and the potential benefits beyond the current COVID-19pandemic-related restrictions [1]. Multiple e-learning formats have been introduced for medical students and ophthalmology trainees to ensure the continuity of ophthalmic education [2, 3]. Moreover, virtual tools and simulation models can allow a controlled and plannable practice of surgical skills, respecting the social distancing [4]. In this light, we describe a virtual corneal suturing dry lab, adopted for medical students and junior registrars at the University of Liverpool, UK. Each student's workstation (also placed at home) is equipped with a desk (surgical table), $5 \times$ highresolution binocular loupes, two eye models (OKULO Anterior Segment BR8 and KERATO models, BIONIKO, Miami, USA), a corneal suturing kit (Hoskins forceps, tying forceps, Vannas scissors, Barraquer suture holder, 10-0 Nylon suture packs) and an IPEVO VZ-R Dual-Mode 8MP document camera connected to a laptop computer (Fig. 1). A 30-min pre-recorded lecture regarding eye anatomy, corneal suturing technique and including video tutorials on cardinal sutures for penetrating keratoplasty, is followed by a remote 30-min live session via Zoom in which the students can ask questions, make attempts at suturing with remote proctoring and the instructor can perform a demonstration of corneal suturing. The trainees can then access the prerecorded lecture through a link and practice independently. After 1 week, the trainees hold a 15-min test in which they perform a maximum of eight sutures on the models, with additional feedback. This performance is recorded to assess "respect for tissue", "time and motion", "instrument handling", and "suturing flow", whereas a photograph of the final result is analyzed with Fiji Image $J$ to calculate mean spacing, width, radiality, and symmetry of sutures.

We propose this virtual dry lab model as a feasible teaching format for microsurgical skills. As the repeated pandemic has led to the interruption of surgical training with potential consequences on their performance [5], a virtual simulation model might help trainees to retain surgical skills.

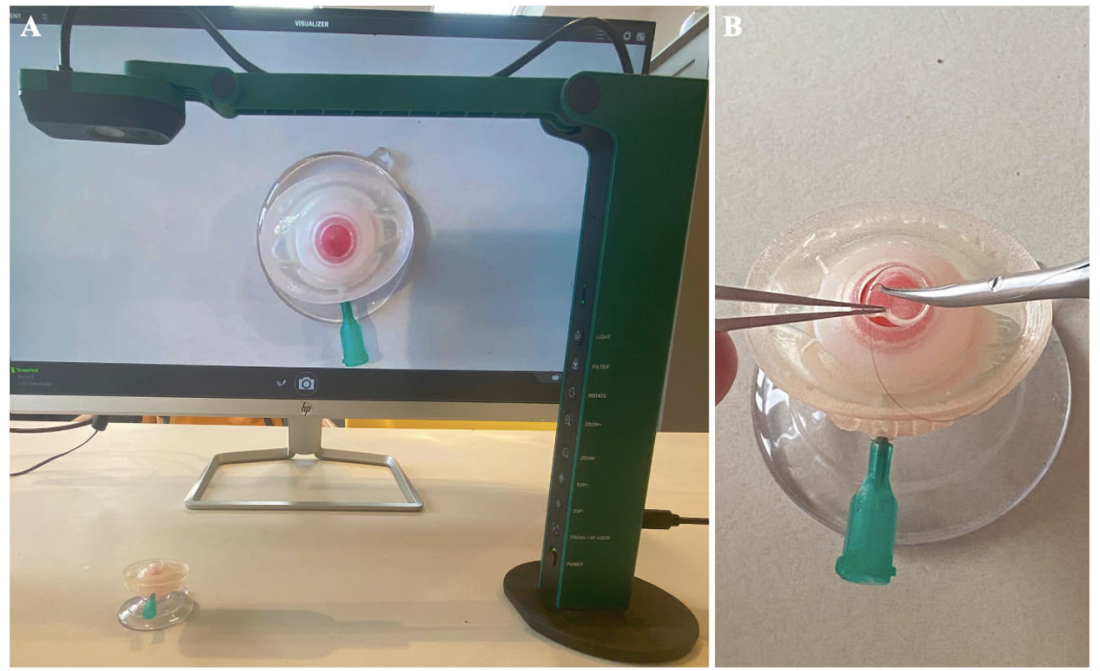

Fig. 1 Corneal suturing dry lab. A Setup of the workstation for the dry lab. B View of the surgical field on the screen. 
Mariantonia Ferrara $\mathbb{D}^{1 凶}$, Mohammed Tanvir Shah ${ }^{2}$, Hannah J. Levis ${ }^{2}$ and Vito Romano (D) ${ }^{2,3}$

${ }^{1}$ Newcastle Eye Centre, Royal Victoria Infirmary, Newcastle Upon Tyne, UK. ${ }^{2}$ Department of Eye and Vision Science, Institute of Life Course and Medical Sciences, University of Liverpool, Liverpool, UK. ${ }^{3}$ Department of Corneal and External Eye Diseases, St Paul's Eye Unit, Royal Liverpool University Hospital, Liverpool, UK. 凶email: mariantonia.ferrara@gmail.com

\section{REFERENCES}

1. He B, Tanya SM, Sharma S. Overcoming barriers in access to ophthalmic education with virtual learning. Eye. 2020. https://doi.org/10.1038/s41433-020-01337-5. Online ahead of print

2. Succar T, Beaver HA, Lee AG. Impact of COVID-19 pandemic on ophthalmology medical student teaching: educational innovations, challenges, and future directions. Surv Ophthalmol. 2021;50039-6257:00098-9.

3. Romano MR, Ferrara M, Rocha-de-Lossada C, Angi M, Govetto A, Mastropasqua R, et al. Active e-learning in ophthalmology through live webinars: back to the theatre. Eye. 2020. https://doi.org/10.1038/s41433-020-01239-6. Online ahead of print.

4. Lee R, Raison N, Lau WY, Aydin A, Dasgupta P, Ahmed K, et al. A systematic review of simulation-based training tools for technical and non-technical skills in ophthalmology. Eye. 2020;34:1737-59.

5. Ferrara M, Pagano L, Kadhim MR, Romano D, D'Alterio FM, Coco G, et al. Comment on: Impact of reduced elective ophthalmic surgical volume on U.S. hospitals during the early COVID pandemic. J Cataract Refract Surg. 2021:47:1103-4.

\section{AUTHOR CONTRIBUTIONS}

$\mathrm{VR}, \mathrm{HJL}$, and MF contributed to the conception of the work. MTS contributed to acquiring the data. MTS and MF contributed to writing the first draft. VR created Fig. 1. VR and HJL provided feedback on the draft. All authors revised the final version. All authors read and approved the final paper. All authors agree to be accountable for all aspects of the work.

\section{COMPETING INTERESTS}

The authors declare no competing interests.

\section{ADDITIONAL INFORMATION}

Correspondence and requests for materials should be addressed to Mariantonia Ferrara.

Reprints and permission information is available at http://www.nature.com/ reprints

Publisher's note Springer Nature remains neutral with regard to jurisdictional claims in published maps and institutional affiliations. 